\title{
Developmental switches that hold the key to a revolution in crop biotechnology
}

\section{Rayasandram Muniyappa Ranganath}

Kaufmann et al. (Regulation of transcription in plants: mechanisms controlling developmental switches. Nature Rev. Genet. 11, $830-842(2010))^{1}$ have provided valuable insights into transcriptional regulation during well-known developmental switches in Arabidopsis thaliana - including the development of the inflorescence meristem from the apical meristem and the establishment of floral organ identities. Similar intensive studies of the developmental switches in the angiosperm nucellus are urgently needed to improve understanding of developmental reprogramming towards apomixis; characterizing the regulation of apomixis could lead to important biotechnological applications.

A nucellar cell normally undergoes meiosis to produce a haploid female gametophyte (FG, which includes the egg) during angiosperm sexual reproduction. In apomictic angiosperms, nucellar cells can avoid meiosis to produce an unreduced, diploid FG or even directly transdifferentiate into a somatic embryo (adventitious embryony) and eventually produce a clone. Manipulating these apomictic developmental switches to produce 'clones-throughseeds' has been a cherished objective in crop biotechnology ${ }^{2}$. As Kaufmann et al. discuss, transcriptional regulators are known to initiate correct developmental transitions or cell fate specifications by coordinating the expressions of required genes ${ }^{1}$. However, little is known about these aspects of apomictic developmental transitions.
For agriculture, the aim would be to produce asexual/adventitious embryos with genetic stability through generations. To achieve this, greater understanding of regulatory processes is required. Genetic variation among the progeny could be avoided by eliminating or suppressing meiotic recombination or meiosis itself. Although basic meiotic processes are conserved among species, diverse mechanisms control the mitosis-to-meiosis transition ${ }^{3}$. For example, the genes IME1 and IME2 in budding yeast, mei2 in fission yeast, stimulated by retinoic acid 8 (Stra8) in mice and ameioticl (am1) in maize regulate the initiation of meiosis in different ways ${ }^{3}$. In $A$. thaliana, mutants in which female meiosis is abolished - such as dyad (also known as swi1) mutants ${ }^{4,5}$, spo 11 rec 8 osd 1 triple mutants ${ }^{6}$ or ago 9 mutants that fail to silence a transposable element-derived small RNA pathway ${ }^{7}-$ produce a functional but unreduced, diploid FG. Elimination of meiosis, however, may increase ploidy of both the embryo and endosperm over successive generations, and the implication of this for crop productivity is uncertain. Another approach that has been proposed is chromosome engineering to suppress meiotic recombination by using balancer chromosomes carrying localized inversions ${ }^{8}$.

A challenge in using adventitious embryony is how to combine it with a normally developed endosperm, either through fertilization (single fertilization) or autonomous development. The molecular mechanisms that underlie the developmental switch that directly transforms a somatic plant cell to an embryo are still elusive ${ }^{9}$. Several genes, such as WUSCHEL (WUS), SOMATIC EMBRYO RECEPTOR-LIKE KINASE 1 (SERK1), LEAFY COTYLEDON 1 (LEC1), LEC2 and $B A B Y B O O M(B B M)$, can induce embryonic growth in plant somatic cells ${ }^{10}$, and Polycomb group proteins are also implicated in somatic embryogenesis ${ }^{11}$. However, it is uncertain whether these pathways for reprogramming of plant somatic cells towards embryogenesis are conserved among species.

Given their immense biological and biotechnological significance, apomictic developmental switches deserve greater attention in future research.

Rayasandram Muniyappa Ranganath is at the Cytogenetics and Developmental Biology Laboratory, Department of Botany, Bangalore University, Jnanabharathi 560056, India. e-mails: rayasran@rediffmail.com; ranganath@bub.ernet.in

Published online 1 February 2011

Kaufmann, K. et al. Regulation of transcription in plants: mechanisms controlling developmental switches. Nature Rev. Genet. 11, 830-842 (2010).

2. Spillane, C. et al. Apomixis technology development virgin births in farmers' fields. Nature Biotech. 22, 687-691 (2004)

3. Pawlowski, W. P. et al. Maize AMEIOTIC1 is essential for multiple early meiotic processes and likely required for the initiation of meiosis. Proc. Natl Acad. Sci. USA 106, 3603-3608 (2009).

4. Agashe, B. et al. Identification and analysis of DYAD: a gene required for meiotic chromosome organisation and female meiotic progression in Arabidopsis. Development 129, 3935-3943 (2002).

5. Ravi, M. et al. Gamete formation without meiosis in Arabidopsis. Nature 451, 1121-1124 (2008) d'Erfurth, l. et al. Turning meiosis into mitosis. PLoS Biol. 7, e1000124 (2009).

7. Olmedo-Monfil, V. et al. Control of female gamete formation by a small RNA pathway in Arabidopsis. Nature 464, 628-632 (2010).

8. Chan, S. W. L. Chromosome engineering: power tools for plant genetics. Trends Biotechnol. 28, 605-610 (2010).

9. Vogel, G. How does a single somatic cell become a whole plant? Science 309, 86 (2005).

10. Ranganath, R. M. Harnessing the developmental potential of nucellar cells: opportunities and barriers. Trends Biotechnol. 22, 54-60 (2004).

11. Chanvivattana, Y. et al. Interaction of Polycomb group proteins controlling flowering in Arabidopsis. Development 131, 5263-5276 (2004).

Competing interests statement

The author declares no competing financial interests. 